Research Article

\title{
Neural Network Algorithm MRI Images for Analysis of Influencing Factors for Patellar Dislocation in Exercise
}

\author{
Zhuorong Gao $\mathbb{D}^{1}{ }^{1}$ Guangsheng Wang $\mathbb{D}^{1}{ }^{1}$ and Zhi Zhang $\mathbb{D}^{2}$ \\ ${ }^{1}$ Anhui Medical College, Hefei 230032, Anhui, China \\ ${ }^{2}$ Anhui No. 2 Provincial People's Hospital, Hefei 230032, Anhui, China \\ Correspondence should be addressed to Zhuorong Gao; gaozhuorong@ahyz.edu.cn
}

Received 9 May 2021; Revised 8 June 2021; Accepted 12 July 2021; Published 20 July 2021

Academic Editor: Gustavo Ramirez

Copyright ( 2021 Zhuorong Gao et al. This is an open access article distributed under the Creative Commons Attribution License, which permits unrestricted use, distribution, and reproduction in any medium, provided the original work is properly cited.

The study focused on segmentation effects of the improved algorithm of traditional neural network algorithm, small kernels twopath convolutional neural network (SK-TPCNN) combined with random forest (RF) algorithm on MRI images for patella, and the influencing factors of patellar dislocation during exercise. In this article, the MRI images for patellar dislocation patients were detected by virtue of the neural network algorithm, to establish the patella-related MRI image segmentation algorithm. In terms of dice similarity coefficient (DSC), positive predictive value (PPV), and sensitivity, the detection accuracy of MRI images for patella was evaluated, and the segmentation effect of MRI images for patella was assessed. 30 patients, who were diagnosed as patellar dislocation patients in hospital, were chosen as the research subjects. No matter whether the MRI images of the patients went through the processing of the neural network algorithm or not, all of them were analyzed. The results showed that, among the traditional neural network algorithm, SK-TPCNN algorithm, and SK-TPCNN + RF algorithm, the DSC values were $0.82,0.71$, and 0.79 , respectively; the PPV values were $0.77,0.59$, and 0.85 , respectively; and the sensitivity values were $0.79,0.62$, and 0.89 , respectively. Obviously, the various parameters of the SK-TPCNN + RF algorithm were significantly greater than those of the SKTPCNN algorithm, and the difference was statistically significant $(P<0.05)$. It indicated that the segmentation ability of MRI images for patella of the NN algorithm was clearly improved, and the MRI image resolution was dramatically raised, which provided a referable basis for the MRI diagnosis of patients with patellar dislocation during exercise.

\section{Introduction}

The acute patella dislocation (APD) refers to the patella severely displaced from the femoral trochlear, which are induced by multiple factors, resulting in the loss of articular surface convolution. The chief symptoms include pain, swelling, restricted movement, and joint hydrops of knee joints [1]. The common APD imaging examination methods cover the X-ray, CT, and magnetic resonance imaging (MRI) examinations [2]. As compared with ordinary X-ray and CT examination, the MRI examination is featured by no radiation, multiparameter imaging, and high resolution for soft tissue, and it can obtain the different degrees of damage to bone, cartilage, synovium, ligament and other tissues, the extension and degree of the bone contusion, bone marrow edema and osteochondral damage of the patellofemoral joint, and the injury location and extent of the medial retaining ligament and medial collateral ligament of patella through a single scanning. The MRI examination has become the preferred examination method for the diagnosis of APD $[3,4]$.

In recent years, the convolutional neural networks (CNN) have turned into the most popular method in the image classification field. A great number of large-scale labeled available data sets have emerged, and a huge progress has been made in hardware such as graphics processing units (GPUs), driving the improvement of CNN performance and its extensive application in medical image analysis. The CNN-based patella segmentation methods are mainly classified into two types: block-based patella MRI segmentation and Fully Convolutional Networks- (FCN-) based patella MRI segmentation $[5,6]$. Some studies suggest that 
the CCN has achieved a good effect in the MRI diagnosis of Alzheimer's disease, brain tumor, breast cancer, etc. [7-9], but whether the CCN has been currently applied in the diagnosis of MRI images of patients with patellar dislocation during exercise has not been reported yet. In the diagnosis of patella dislocation, the anterolateral femoral condyle contusion usually has higher signals in MRI. The torn ligament, bone contusion, lateral femoral condyle or osteochondral fracture of the inner edge of the patella, and patella tilt/ subluxation can be used as a basis to judge APD [10, 11]. To optimize the imaging effect of MRI images for APD patients, the MRI imaging data of APD patients were collected, and the new NNA-based SK-TPCNN model and integrated model of SK-TPCNN and RF were used in the study, to analyze MRI images and measure the required data. The statistical software was employed to analyze and explore the imaging measurement significance of various anatomical risks in acute skeletal dislocation and analyze the MRI information of patients with acute skeletal dislocation during exercise, so as to raise the early diagnosis efficiency of APD patients and discuss the influencing factors for acute skeletal dislocation, providing imaging basis for the prevention of patellar dislocation during exercise and the selection of clinical treatment plans.

\section{Materials and Methods}

2.1. Subjects and Grouping. 30 confirmed PH patients, who received the right cardiac catheterization in hospital from June 2019 to September 2020, were chosen as subjects, and all patients underwent MRI examination, including 14 male patients and 16 female patients. The age of patients ranged from 16 to 47 years old, and the average age was $23.62 \pm 7.35$ years old. Among all the patients, 18 patients had patella dislocation in their left knees, while 12 patients had patella dislocation in their right knees. Inclusion criteria are as follows: (1) 30 selected patients who underwent the injury examination in 21 days; (2) the MRI showed that the APD patients got patella dislocation; (3) the patients who had a history of instant reset for patella dislocation, which was found in outpatient medical record; (4) the patients who went through other operations in addition to recurrent patella dislocation, combined collateral ligament injury, or knee surgery. Exclusion criteria are as follows: (1) the patients who got skeleton dislocation and received the knee joints operation; (2) the patients who suffered from cruciate ligament rupture or severe fracture of fat bone, femur, or embryonic bone; (3) the patients with complicated gonarthritis; (4) the patients who were not able to keep improper positions during examination and obtained bad-quality images. The procedure was approved by the Ethics Committee of our hospital, and all the subjects who were included into the study signed the informed consent form.

\subsection{Establishment of NNA-Based Target Patella Detection} Model. Based on the basic structure of the CNN model (as shown in Figure 1), the MRI images for patella dislocation of the patients were processed. The specific process is shown as follows: the original pixels of input images are acquired through the CNN, transformed by the convolutional layer, nonlinear activation function, and the pooling layer, and then entered the fully connected layer (FC) to assign the number of categories or probability. The input category served as the category with the highest probability. The CCN accomplished various analyses of images by means of these featured conversion layers.

The weights and deviations of all the layers in the CNN structure were optimized by means of minimizing the loss function. First, the training data set was entered into the CNN network and, next, passed through various linear and nonlinear transformation layers for forward propagation; after that, the Softmax was used to transform the output features into the predicted and labeled probability distribution. Finally, the backpropagation was used to optimize algorithm, minimizing the loss function and its training process, as shown in Figure 2; the patella segmentation model based on the traditional CNN algorithm is shown in Figure 3:

\subsection{Establishment of SK-TPCNN-Based Patella Segmentation} Model. The two-path convolutional neural network model (SK-DPCNN) with big and small convolutional kernel paths was established for PD patients based on the effective SKTPCNN model, which was verified in the literature [12]. Two paths include the local paths and global paths. The Brats 2015 training set was used in training, while the cross entropy served as the loss function and can be used to measure the difference between the real distribution and the predicted distribution, which is defined as follows:

$$
K\left(k, k^{*}\right)=\sum_{a \in \text { voxels }} \sum_{b \in \text { classes }} k_{a, b} \log \left(k_{a, b}^{*}\right)
$$

where $a, b$ represent small convolutional kernel path and large convolutional kernel path, respectively, $k^{*}$ indicates the predicted probability distribution, and $k$ represents the real distribution. The smaller cross entropy means that two probability distributions are much closer. Because the loss function is highly nonlinear, Adam algorithm with adaptive moment was adopted as the optimized algorithm, in order to calculate the adaptive learning rates of different parameters and ascertain the parameter values.

\subsection{Establishment of Patella Segmentation Model Based on} SK-TPCNN +RF-Integrated Models. The postprocessing operations are completed with Random forest (RF) as a classifier and the SK-TPCNN algorithm as a feature extraction unit, to effectively integrate HD redundant features, determine the types of pixels in the images for patella region, and improve the classification result of SK-TPCNN model. RF refers to an integrated learning method for classification and regression tasks. It can be used to integrate a certain number of decision trees, which are independent of each other. The randomness in training samples and construction of decision trees is selected to reduce the error rate and avoid the occurrence of overfitting. The mathematical expression of the decision tree is shown as follows: 


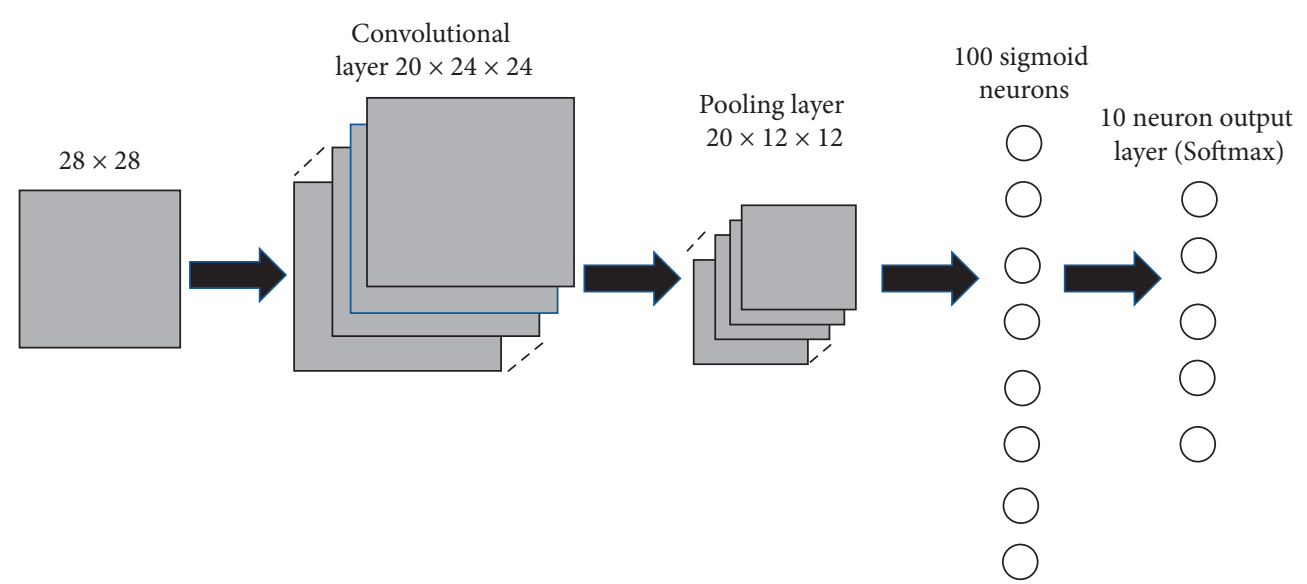

Figure 1: Model diagram for CNN.

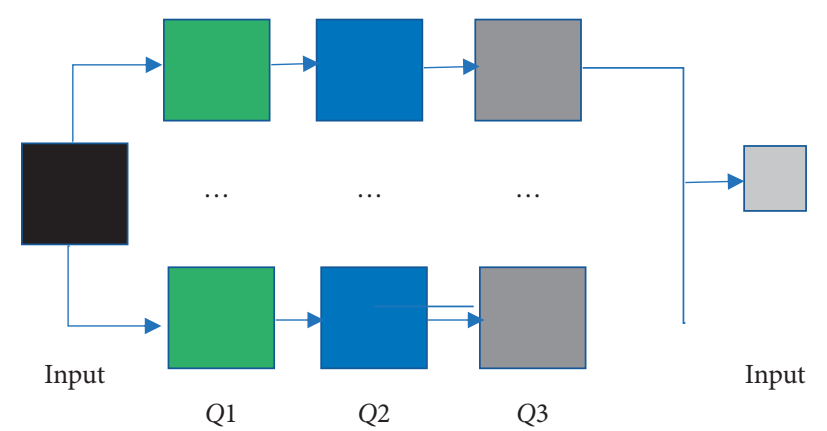

FIgURE 2: Training process of CNN model.
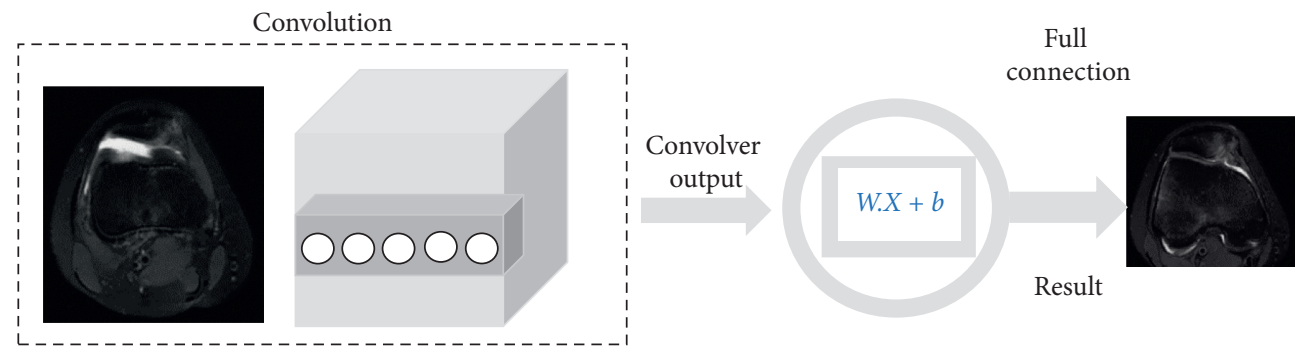

Figure 3: Segmentation framework of CNN-based patella images.

$$
\left\{k\left(z, \beta_{n}\right), \quad n=1,2, \ldots, n \text { tree }\right\}
$$

In equation (2), $k\left(z, \beta_{n}\right)$ represents the decision tree, $z$ indicates the test data set, and $\beta_{n}$ is the training set of independently distributed decision trees, which determines the generation process of a tree. The final classification result depends on the votes of a decision tree. The specific algorithm steps of RF include (1) data sampling; (2) generation of a decision tree; (3) classification of votes. Among them, the nodes splitting of the decision tree for $M$ sample sets is completed with the Gini index as the basis for the splitting features, and the minimum Gini index is employed for splitting. Provided that $M$ represents the number of sample types in the sample set $S$, the Gini index is shown as follows:

$$
\operatorname{Gini}(S)=1-\sum_{b=1}^{M} P_{b}^{2} \text {. }
$$

In equation (3), $P_{b}$ represents the probability of category $b$, and Gini $(T)$ indicates the probability of two samples with different results randomly drawn from the data set T. Therefore, the smaller the Gini $(S)$ is, the higher the purity of the data set $S$ is. The adequate merisis of leaf nodes in each decision tree is proceeding until its minimum value of impurity is reached. The randomness in sampling can effectively prevent occurrence of overfitting; therefore, the pruning process is not required in the generation of decision trees. The well-trained RF is tested in the classification of 
votes. The test data $T$ is input into the RF and passes through all the trees to a certain leaf node. The probability that the sample $T$ belongs to the category $V$ is shown as

$$
P(V \mid T)=\sum_{r=1}^{R} P(V \mid T)
$$

In equation (4), $R$ indicates the number of decision trees in the forest, while $P(V \mid T)$ represents the distribution of leaf nodes in splitting. The decision for category $T$ is shown as follows:

$$
\begin{aligned}
C & =\arg (\max P(V \mid T)), \\
c & \in\left\{1, \ldots C_{c}\right\} .
\end{aligned}
$$

The final framework diagram for optimized algorithms of SK-TPCNN and RF is shown in Figure 4:

2.5. Indicators of Evaluating the Segmentation of the NNAProcessed MRI Images. In this article, in terms of DSC, Positive Predictive Value (PPV), and Sensitivity, three tumor areas including complete area, core area, and enhancing area for patellar dislocation were measured, provided that $X$ represents the real patellar dislocation area segmented by an expert, $\mathrm{Y}$ indicates the patellar dislocation area predicted by the algorithm, and the DSC can reflect the similarity level between the predicted result and the expert's segmentation label. The DSC is calculated by overlapping the predicted segmentation result $P$ and the expert's segmentation label $G$; the Sensitivity is to measure the accuracy of correctly classified tumor labels; the PPV represents the proportion of all correctly segmented patellar dislocation points in the predicted patellar dislocation points, which are defined as follows:

$$
\begin{aligned}
\text { DSC } & =\frac{|X \cap Y|}{(|X| \cup|Y|) \div 2}, \\
\text { Sensitivity } & =\frac{|X \cap Y|}{|X|}, \\
\text { PPV } & =\frac{|X \cap Y|}{|Y|} .
\end{aligned}
$$

2.6. Analysis on Correlation of Influencing Factors for Patellar Dislocation during Exercise. It was stated in a flood of literatures that the patellar dislocation was intimately correlated with the anatomical risks in the patellofemoral joints. Currently, the highly recognized anatomical risks of acute patella dislocation involve patella alta, trochlear dysplasia, tibial tubercle-trochlear groove (TT-TG), out-shifted dermal tuberosity, lateral patella tilt, and rotational deformity.

The NNA-based MRI sagittal images of the patients were used, and the ISI measurement methods were adopted to measure the ratio of the length of the patellar tendon to the maximum diameter of the patella. If the ratio is greater than 1.3 , it is identified as the patella alta; the femoral trochlea is classified into different types as per the classification standard put forward by Dejour. H. The normal femoral trochlea

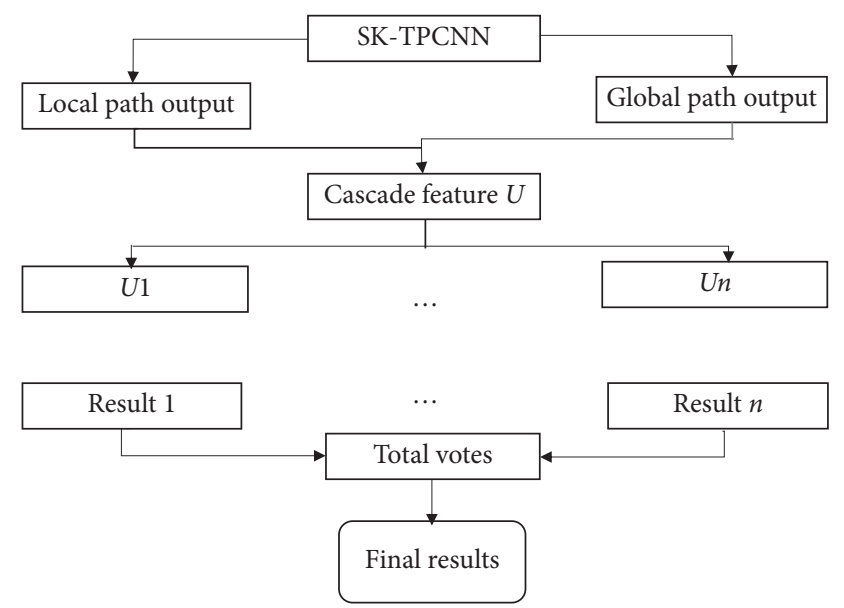

FIGURE 4: Structure of the optimized model from SK-TPCNN and RF.

is defined as type A, the trochlea with shallow intermediate sulcus (the concave angle of femur trochlear is $>145^{\circ}$ ) is identified as type $B$, the trochlea without intermediate sulcus is defined as type $C$, the trochlea with higher outer surface or lower inner surface is confirmed as D type, and the trochlea with abnormal protrusion between the inner and outer surfaces is identified as type E. The postprocessing image superposition technology is used to superimpose the two scanning layers and measure the TT-TG distance; the patella tilt angle (PTA) refers to the angle between the extension line with the maximum transverse diameter at the tangential position of the patella and the bare femur. If the angle is $>20^{\circ}$, it is regarded as positive; the lateral patella tilt angle (LPTA) in the MRI images of the patients was calculated. When the measured value is $\leq 0^{\circ}$, it indicates the patella tilt. The positive reference values of all the measured data are shown in Table 1.

2.7. Statistical Method. The SPSS19.0 statistical software was used to process experimental data, and the mean value of measurement data \pm the standard deviation was expressed as $\bar{x} \pm s$. The comparison of mean values of all groups was verified by $t$. The measurement data was expressed by percent (\%) and tested by $\chi^{2}$, and $P<0.05$ indicates the statistical significance in differences.

\section{Results}

3.1. Features of MRI Images for APD Patients in Normal Conditions. Figure 5 is an MRI image of a 16-year-old female with APD. The patient's femoral trochlear was shallow, and there was injury to medial patella bone and its cartilage, accompanied by contusion of the lateral femoral condyle. The patella medial retinaculum exhibited thickened MRI scan signals at the stop point of the patella.

3.2. Features of the NNA-Based MRI Images for PD Patients. Figure 6 shows the segmentation results in the two scenarios. Figure 6(a) presents the original images of T1 
TABLE 1: Positive reference value of measured data.

\begin{tabular}{lr}
\hline & Positive reference value \\
\hline ISI & $>1.3$ \\
Osteoarthritis FTA & $\geq 143^{\circ}$ \\
Cartilaginous FTA & $\geq 145^{\circ}$ \\
Tendinous-cartilaginous TT-TG & $\geq 20$ min \\
LPTA & $\leq 0^{\circ}$ \\
\hline
\end{tabular}

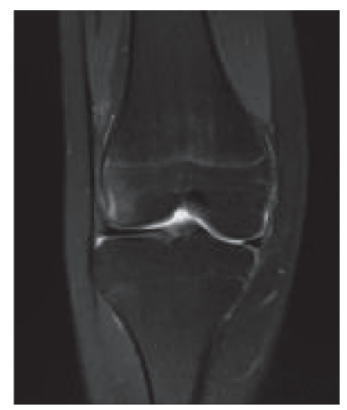

(a)

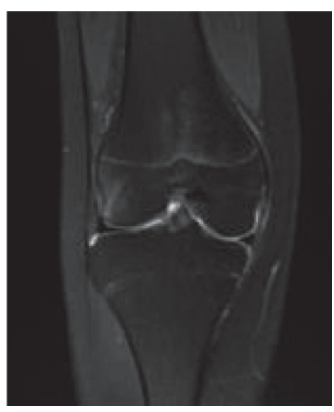

(b)

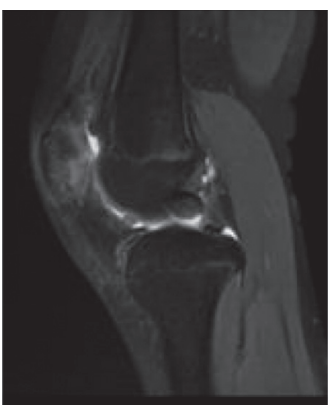

(c)

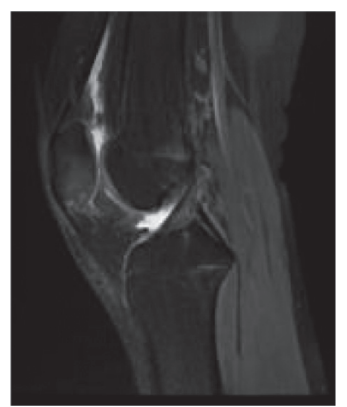

(d)

FIgURE 5: The MRI image of an APD patient. (a, b) The coronal MRI images of the patient's knee. (c, d) The T2W1-phase MRI images of the patient.

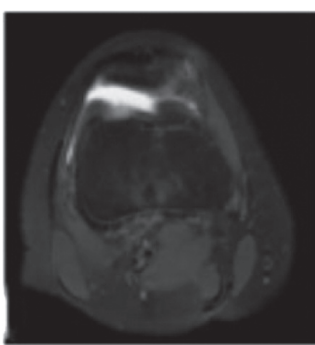

(a)

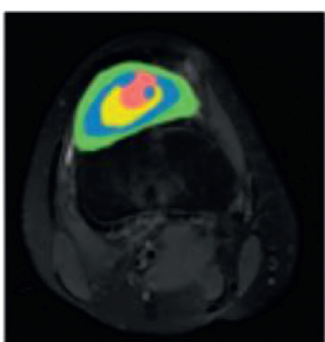

(b)

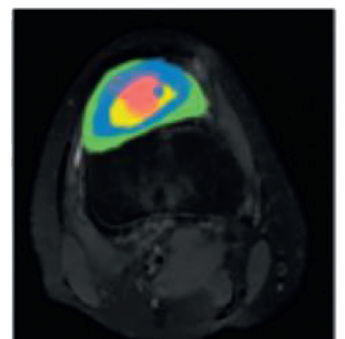

(c)

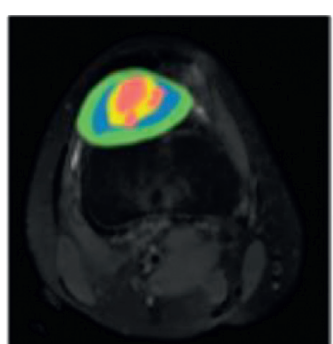

(d)

Figure 6: Comparison of segmentation of different methods. (a) The patient's T1 model imaging. (b) The label image segmented by an expert. (c) The segmentation result of the SK-TPCNN model. (d) The segmentation result of the SK-TPCNN + RF model. The red represents the necrotic area, and the green indicates the edema area. The blue stands for the nonenhanced patella dislocation area, and the yellow indicates the enhanced patella area.

model. Figure 6(b) reveals the area segmentation labels marked out by experts. Figure 6(c) displays that the SKTPCNN model achieved relatively accurate segmentation results and had strong feature extraction capabilities, to effectively integrate the features of two paths. The images for the enhanced tumor area had the best segmentation effect; however, the images for other areas were relatively rough; for instance, the images for edema area had the oversegmentation signs. Figure 6(d) shows that the output features of SK-TPCNN were classified by the RF classifier in terms of pixels, and the performance was dramatically improved, and the segmentation edge for each tumor area became more delicate. The above comparison experiments manifested that SK-TPCNN had good feature extraction capabilities. RF and SK-TPCNN can be integrated to highly raise the accuracy of classification and achieve the precise segmentation for patella dislocation.

\subsection{Comment on Quality of the NNA-Based MRI Images.} Figure 7 shows image quality of different algorithms in different regions. As shown in Figure 7, among the traditional neural network algorithm, SK-TPCNN algorithm, and SK-TPCNN + RF algorithm, the DSC values were $0.82,0.71$, and $0.79, \mathrm{PPV}$ values were $0.77,0.59$, and 0.85 , respectively, and sensitivity values were $0.79,0.62$, and 0.89 , respectively. Obviously, various parameters of the SK-TPCNN + RF algorithm were significantly higher than those of the SK-TPCNN algorithm, and the difference was statistically significant $(P<0.05)$. 


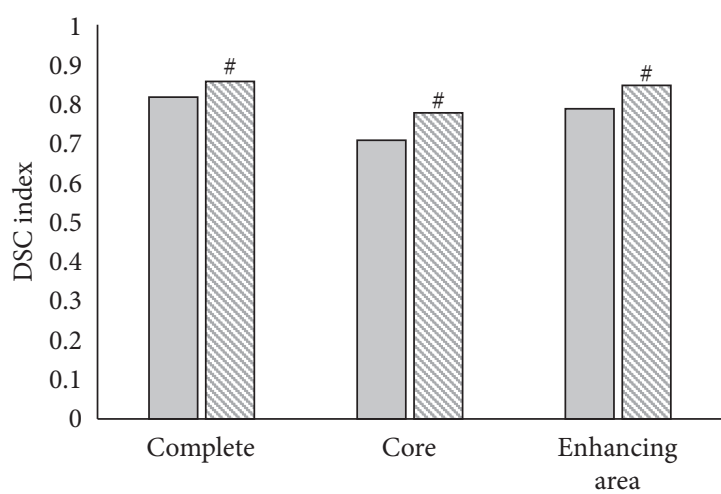

$\square$ SK-TPCNN

\& SK-TPCNN + RF

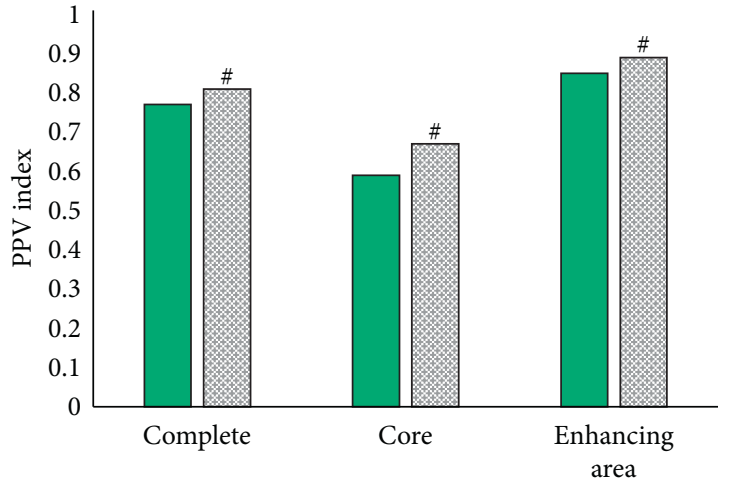

a SK-TPCNN

圆 SK-TPCNN $+\mathrm{RF}$

(a)

(b)

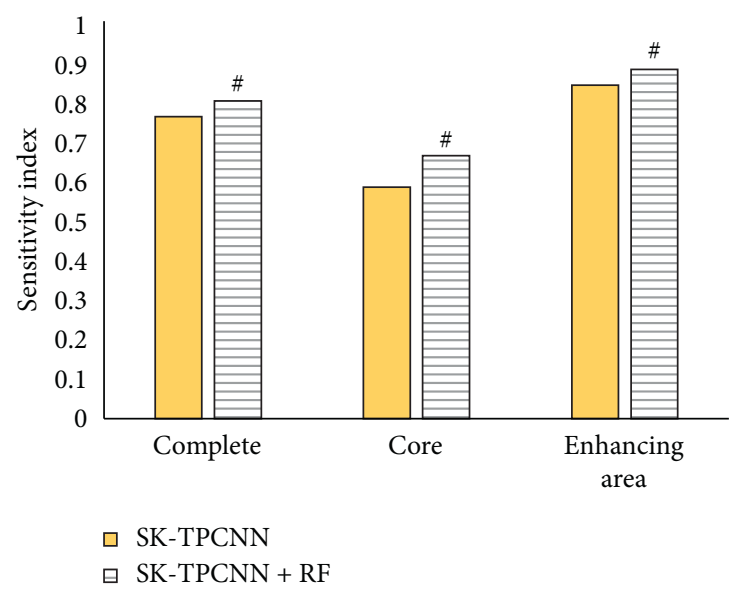

(c)

FIgURE 7: Image quality of different algorithms in different regions. (a) The DSC value. (b) The PPV value. (c) The sensitivity value. \# indicates statistically significant differences compared with the SK-TPCNN algorithm, $P<0.05$.

\subsection{Analysis of Risk Factors of NNA-Based MRI Images for} APD Patients. The case data and MRI images for APD patients were analyzed. The patients with ISI $>1.3$ were identified as high patella patients, the patients with femoral trochlear depth $>3 \mathrm{~mm}$ were defined as femoral trochlear dysplasia, and those with skeletal TT-TG distance $>15 \mathrm{~mm}$ were diagnosed as displacement of embryonic bone nodules, while those with lateral trochlear inclination angle $\leq 0^{\circ}$ were identified as the patella tilt. Figures 8 and 9 show the frequency of the four anatomical risk factors in all APD patients and their percentages in each group, which were stated in literatures. It showed that, among the 30 patients, 5 had high patella, 8 had femoral trochlear dysplasia, 7 had tibial joint displacement, and 10 had patella tilt. And their proportions were $16.7 \%, 26.7 \%, 23.3 \%$, and $33.3 \%$, respectively.

\section{Discussion}

A great number of studies manifested that the patella dislocation arising from exercise had a higher proportion in the clinical APD patients. When the femur in motion is subject to internally rotated external force relative to the tibia, the

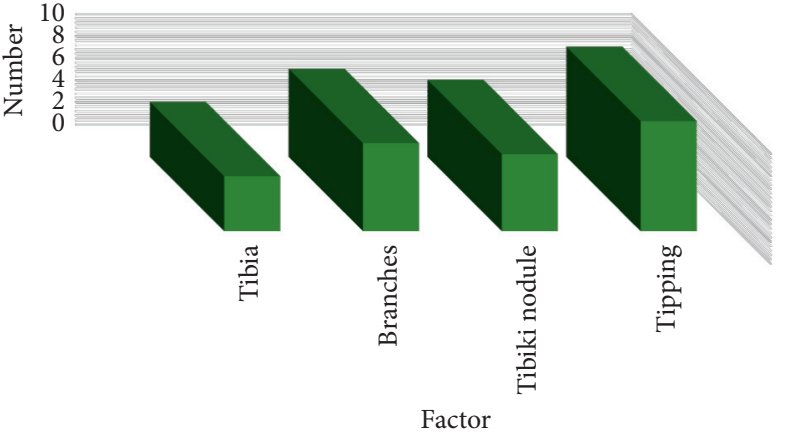

FIGURE 8: Frequency of risk factors in APD patient.

inner surface of the patella bumps into the lateral femoral condyle, and the stress to cortical bone is transmitted to the bone trabecula, resulting in congestion, edema, and hemorrhage of trabecular bone. The different degrees of damage to the trabecular bone occurred due to external stress, and the patella was instantly separated from the trochlear groove, leading to patella dislocation [13-15]. The individual differences, and noise and artifacts in MRI images, and other 


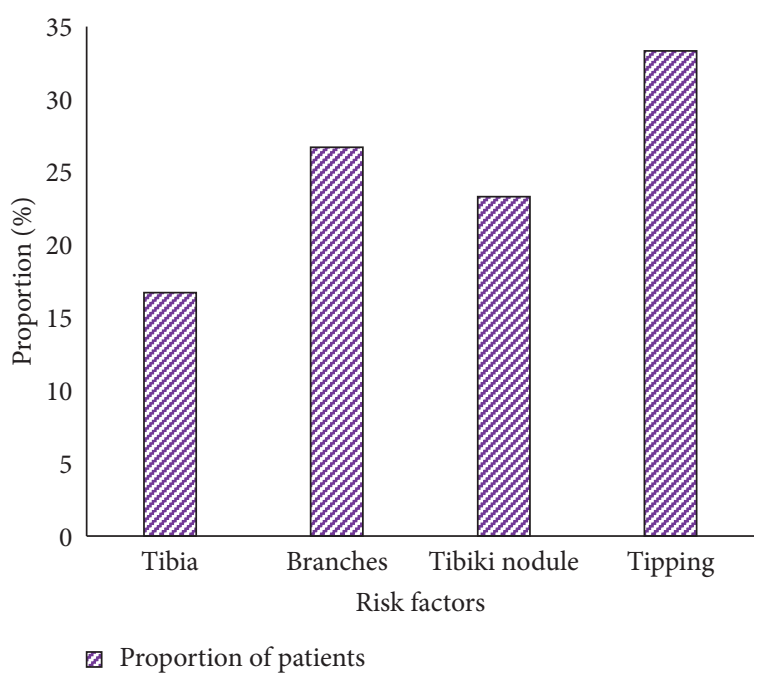

FIgURE 9: Proportion of risk factors in APD patients.

interference factors $[16,17]$, resulted in a certain probability of missed diagnosis in the diagnosis of clinical APD patients. The neural network algorithms were adopted to process MRI images, which was applicable to MRI images for the patella with relatively complicated structures [18].

Based on optimized algorithms for patella segmentation of the traditional CNN models, a new type of SK-TPCNN model was proposed in this article. By virtue of the advantages of the SK-TPCNN algorithm, it can be used to correct the information about the patella MRI image sequence of patient, synchronously extract multiple features, and enhance the image feature extraction ability. It achieved a good effect in segmentation for MRI images. RF can be used as a postprocessing program to build an integrated optimal model. Some studies showed that the DSC value, PPV value, and image sensitivity of MRI image of the patient under the SK-TPCNN + RF algorithm model were extensively applied in verification of the effect of $3 \mathrm{D}$ medical images segmentation $[19,20]$, which can depict the segmentation effect more vividly. The research results in this article manifested that the SK-TPCNN algorithm can converge faster and achieve lower loss values, reduce the calculation amount, improve the abstract characterization ability, obtain higher fitting effects, to enhance the clarity of MRI imaging, and raise the diagnosis efficiency of APD; the integrated optimal model of SK-TPCNN and RF improved the segmentation results. The parameter values for the MRI images processed through the SK-TPCNN + RF algorithm were obviously higher than those of the pure SK-TPCNN algorithm, of which the substantial differences $(P<0.05)$ had statistical significance. The SK-TPCNN $+\mathrm{RF}$ algorithm possessed the segmentation performance for enhanced area and increased the accuracy in the diagnosis of acute patellar dislocation. However, this study still has some weaknesses. For instance, this article has fewer samples of MRI image data of APD patients, and less convincible evaluation on risk factors, insufficient evaluation factors in the assessment of NNA-based MRI images, which will be further explored in a later work. In conclusion, the feature extraction ability of
NNA-based MRI images for patella edge was enhanced, and the segmentation ability was greatly strengthened, which provided a referable basis for the diagnosis and treatment of PD patients.

\section{Conclusion}

In the study, the SK-TPCNN and RF algorithm were integrated and upgraded based on the traditional neural network algorithms, to provide greater soft tissue contrast resolution for MRI images. Its DSC value, PPV value, and image sensitivity indexes were apparently superior to the single SK-TPCNN model. It can explicitly display the bone contusion, joint hydrops, and other features, to confirm injuries of cartilage and ligament structure and more accurately assess the potential pathogenesis and enhance the extraction ability of patella edge features, and the segmentation ability. It can identify the misdiagnosis arising from detailed deviation in traditional MRI diagnosis and reduce the missed diagnosis rate, which provided a theoretical basis for improving the clinically used MRI diagnosis and treatment technology for PD patients. However, due to the complicated mechanism of patellar dislocation during exercise, a unified conclusion was not reached in many relevant studies, and the influencing factors of patellar dislocation have not been studied further in terms of MRI imaging data and will be explored further in the future.

\section{Data Availability}

No data were used to support this study.

\section{Conflicts of Interest}

The authors declare that they have no conflicts of interest.

\section{Authors' Contributions}

Zhuorong Gao and Guangsheng Wang contributed equally to this work.

\section{References}

[1] D. L. Lyu, D. B. Wang, H. Shao, Y. Zhu, and D. Zhang, "Application of MRI in diagnosis of transient dislocation of patella," Zhong Guo Gu Shang, vol. 31, no. 6, pp. 577-581, 2018, in Chinese.

[2] T. L. Hsu, S. M. Lin, C. H. Chang, and T. Y. Lan, "Neglected pediatric osteochondral fracture dislocation of the patella," Case reports in orthopedics, vol. 2019, Article ID 2904782, 2019.

[3] S. Höhne, K. Gerlach, L. Irlenbusch, M. Schulz, C. Kunze, and R. Finke, "Patella dislocation in children and adolescents," Zeitschrift für Orthopädie und Unfallchirurgie, vol. 155, no. 2, pp. 169-176, 2017.

[4] D. Saragaglia, J. J. Banihachemi, and R. Refaie, "Acute instability of the patella: is magnetic resonance imaging mandatory?" International Orthopaedics, vol. 44, no. 11, pp. 2299-2303, 2020.

[5] W. Qiu, D. Li, X. Jin, F. Liu, and B. Sun, "Deep neural network inspired by iterative shrinkage-thresholding algorithm with 
data consistency (NISTAD) for fast undersampled MRI reconstruction," Magnetic Resonance Imaging, vol. 70, pp. 134-144, 2020.

[6] X. Han, "MR-based synthetic CT generation using a deep convolutional neural network method," Medical Physics, vol. 44, no. 4, pp. 1408-1419, 2017.

[7] S. Basaia, F. Agosta, L. Wagner et al., "Automated classification of Alzheimer's disease and mild cognitive impairment using a single MRI and deep neural networks," NeuroImage: Clinica, vol. 21, Article ID 101645, 2019.

[8] S. Pereira, A. Pinto, V. Alves, and C. A. Silva, "Brain tumor segmentation using convolutional neural networks in MRI images," IEEE Transactions on Medical Imaging, vol. 35, no. 5, pp. 1240-1251, 2016.

[9] R. Ha, S. Mutasa, J. Karcich et al., "Predicting breast cancer molecular subtype with MRI dataset utilizing convolutional neural network algorithm," Journal of Digital Imaging, vol. 32, no. 2, pp. 276-282, 2019.

[10] K. Małecki, K. Pruchnik-Witosławska, D. Gwizdała, P. Grzelak, P. Flont, and K. R. Niedzielski, "Clinical results and MRI evaluation of patellar osteochondral fracture fixation following patellar dislocation," BioMed Research International, vol. 2019, Article ID 7943636, 6 pages, 2019.

[11] M. N. Alosaimi, M. M. Almutairi, S. M. Alshahrani, M. N. Alqahtani, and A. S. Alghamdi, "Osteochondral fracture of the patella without soft tissue injury and with no dislocation: a case report," International Journal of Surgery Case Reports, vol. 78, pp. 48-53, 2021.

[12] L. Sun, Z. Fan, X. Ding, Y. Huang, and J. Paisley, "Region-ofinterest undersampled MRI reconstruction: a deep convolutional neural network approach," Magnetic Resonance Imaging, vol. 63, pp. 185-192, 2019.

[13] H. Purmehdi, A. R. Hareendranathan, M. Noga, and K. Punithakumar, "Right ventricular segmentation from MRI using deep convolutional neural networks," in Proceedings of the 2019 41st Annual International Conference of the IEEE Engineering in Medicine and Biology Society (EMBC), pp. 4020-4023, Berlin, Germany, July 2019.

[14] B. Jiang, C. Qiao, Y. Shi et al., "Evaluation of risk correlation between recurrence of patellar dislocation and damage to the medial patellofemoral ligament in different sites caused by primary patellar dislocation by MRI: a meta-analysis," Journal of Orthopaedic Surgery and Research, vol. 15, no. 1, p. 461, 2020.

[15] H. Cao, Q. An, B. Gou et al., "A new classification of injury patterns of the medial patellofemoral ligament after acute lateral patella dislocation detected using magnetic resonance imaging studies," Injury, vol. 50, no. 2, pp. 534-540, 2019.

[16] S. Li, J. Zhou, D. Liang, and Q. Liu, "MRI denoising using progressively distribution-based neural network," Magnetic Resonance Imaging, vol. 71, pp. 55-68, 2020.

[17] S. S. Gurbani, E. Schreibmann, A. A. Maudsley et al., "A convolutional neural network to filter artifacts in spectroscopic MRI," Magnetic Resonance in Medicine, vol. 80, no. 5, pp. 1765-1775, 2018.

[18] F. Liu, A. Samsonov, L. Chen, R. Kijowski, and L. Feng, "SANTIS: sampling-augmented neural neTwork with incoherent structure for MR image reconstruction," Magnetic Resonance in Medicine, vol. 82, no. 5, pp. 1890-1904, 2019.

[19] F. Hoseini, A. Shahbahrami, and P. Bayat, "AdaptAhead optimization algorithm for learning deep CNN applied to MRI segmentation," Journal of Digital Imaging, vol. 32, no. 1, pp. 105-115, 2019.
[20] F. Guo, M. Ng, M. Goubran et al., "Improving cardiac MRI convolutional neural network segmentation on small training datasets and dataset shift: a continuous kernel cut approach," Medical Image Analysis, vol. 61, Article ID 101636, 2020. 